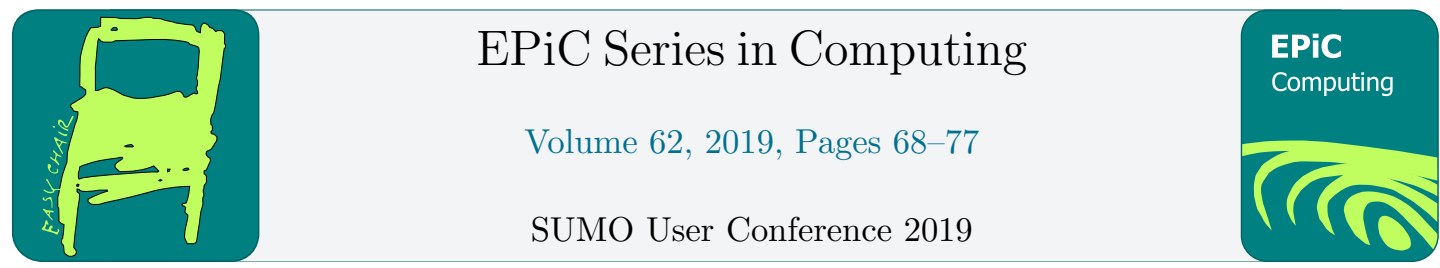

\title{
Investigation of the capacity of train stations in case of a large-scale emergency evacuation
}

\author{
Anna Braun, Mohcine Chraibi, and Lukas Arnold \\ Forschungszentrum Jülich GmbH \\ an.braun@fz-juelich.de, m.chraibi@fz-juelich.de, l.arnold@fz-juelich.de
}

\begin{abstract}
Nature and human-made hazards, like hurricanes, inundations, terroristic attacks or incidents in nuclear power plants, make it necessary to evacuate large urban areas in a short time. So far, the consideration of railway transportation is rarely part of the evacuation strategies. One of the reasons is the unknown capacity of this infrastructure.

In the case of hurricanes Katrina and Rita (USA) the evacuation was accomplished with private vehicles and buses. In Germany, especially in the conurbation of Nordrhein-Westfalen, where many roads are overloaded during the daily rush hours, it will not be possible to use only road dependent vehicles like private cars or busses to evacuate a large number of people into save areas.

After the nuclear power plant disaster of Fukushima, the working group 'AG Fukushima' was founded, which recommends the use of trains for large-scale emergency evacuations. However, it is not clear if the capacity of train stations is enough to handle these large evacuations in time. Hence, this work deals with the question of how the capacity of train stations can be quantified and optimised for this application. In order to estimate the capacity of train stations we use and further develop the Jülich Pedestrian Simulator (JuPedSim), a software for pedestrian dynamics simulations. Therefore, a model of a train station is built in JuPedSim and several parameters like the inflow and outflow of the pedestrians are examined, to find the best routing strategy and organisational actions inside the station. The focus of this contribution lies in the identification of critical bottlenecks. An estimation of which parameters are influencing congestion at these bottlenecks is presented. Additionally, organisational strategies are outlined, which can prevent congestion and increase the capacity of a train station.
\end{abstract}

\section{Introduction}

Nature and human-made hazards like hurricanes, inundations, terroristic attacks or incidents in nuclear power plants make it necessary to evacuate large urban areas in a short time. As many people for multiple reasons do not have access to private vehicles, it is a big challenge for the population and emergency planners to organise enough transportation possibilities in case of a large-scale evacuation. In the literature there are a couple of examples of how to evacuate people by private cars or by buses, see below. However, so far the consideration of railway transportation is rarely part of the evacuation strategies. One of the reasons is the unknown 
capacity of this infrastructure. It is unknown because it is a very complex system as there is no experience with large-scale evacuations using railways. Furthermore, there are very few studies about this topic.

In the USA in case of the hurricanes Katrina and Rita the evacuation was accomplished entirely with private vehicles and buses [1]. However, the evacuation with only private vehicles was hindered by the fact that many people do not have access to private vehicles and were therefore dependant on public buses. The reasons for people having no private vehicles are numerous, see [2]. Moreover, the evacuation with only road dependant vehicles was hindered, because the road capacity is limited. In the case of hurricane Rita the evacuation with private vehicles caused huge congestion, which leads to other problems like accidents, the lack of fuel or broken-down cars [1].

In Neaples (Italy) it exists an evacuation-concept, which uses private vehicles, buses, ships and trains to evacuate people in case of an eruption of the active volcano Vesuvius. It gives a precise evacuation schedule that explicitly defines the evacuation process of the habitats of this region [3]. Therefore the endangered area is divided into three hazard zones, the 'Red Zone', the 'Yellow Zone' and the 'Blue Zone'. The inhabitants of the Red Zone are threatened by pyroclastic flows and ash fall. In the Yellow and Blue Zone the inhabitants are threatened by significant ash fall. Additionally the inhabitants of the Blue Zone are threaten by pyroclastic debris flow and floods. Hence, in case of the evacuation the inhabitants of the Yellow and Blue Zone will be evacuated after the Red Zones inhabitants have been evacuated [3, 4]. Although this is a detailed plan, which has already been tested with more than 1800 inhabitants and volunteers [5], there is still no detailed study about the capacity of the involved train stations.

In Germany, especially in the conurbation of Nordrhein-Westfalen, where many roads are overloaded during the daily rush hours, it will not possible to use only road dependent vehicles to evacuate a large number of people into save areas. After the nuclear power plant disaster of Fukushima, the working group 'AG Fukushima' was founded, which recommends the use of trains for large-scale emergency evacuations [6].

Hence, this work deals with the question of how the capacity of train stations can be quantified and optimised for this application. The capacity of train stations therefore means, how many people could pass the station and enter the trains (save shelters) in a defined time. Consequently, the capacity can be defined as a value [people/hour]. In order to estimate the capacity of a train station in case of a large-scale evacuation, the pedestrian movement inside the station is investigated. Therefore we use and further develop JuPedSim [7]. It is setup a model of a train station in JuPedSim and several parameters like the inflow and leading of the passengers are examined to find the best routing strategy and organisational actions inside the station to increase its capacity.

Unlike other passenger movement simulations, in this study we do not investigate the daily passenger movement of a train station [8] nor an evacuation from a building [9]. Our focus is on the dynamics of passenger movements through a train station under an evacuation context. The difference of these both is, that there is no bi- or multi-directional passenger flow with incoming and outgoing passengers in case of an evacuation. Passengers will only pass through the station and leave by any available train. A difference to an evacuation of a train station (e.g. in case of a fire) is the concentration of the passengers. In an evacuation in case of a fire in the station the concentration of the people would rise the closer they get to the exits. In the evacuations context we consider in this paper, the concentration declines the closer the people get to the train. Additionally the passengers will carry a lot of things, like huge luggage or pets, which they normally would not bring for a journey. The trains will no longer drive by the daily time schedule and they will only head for a few very specified destinations. Therefore the trains 
will arrive at the station empty and will depart with the maximum number of passengers.

\section{Setup and parameters}

In JuPedSim passengers (here called "agents") are modelled as particles with several parameters, like shoulder width, step length or free movement speed, see [10]. In the presented studies the floor-field router [11] and the collision-free speed model for pedestrian dynamics [12] are used. With this model the agents move collision-free e.g. without overlapping other agents or obstacles at any density level. Additionally self-organisational phenomena like lane formation or clogging at bottlenecks can be described. For more information, see [12]. However, the model does not consider group behaviour phenomena like staying together (e.g. families) and herding. Therefore, it is assumed, that passengers who enter the station will act in a rational way. Nevertheless, it is planned to integrate a model for group behaviour phenomena in JuPedSim.

In the presented studies, a simplified model of a train station is used. It corresponds in extension and design to a widespread station type, where the trains can drive through the station in two directions. It consists of an entry hall and a tunnel, which runs under the tracks and has stairs on the right and left to access the platforms, see figure 1. The trains are modelled by doors only and are considered as safe shelters. An agent, who enters one of these doors, will therefore leave the simulation domain.



Figure 1: Simulation setup

As in reality many different types of trains would be used to evacuate the people, the door 
positions can be varied dynamically. Here, the evacuation is considered with a single exemplary train type. Hence, the platform lengths and doors positions are adapted to those of this train type. The relevant dimensions of the train are listed in table 1. To avoid crowding on the platforms, the trains will only approach one of the two tracks of a platform at the same time. By regulating the flow of the doors, different entry times of the trains can be modelled. Here the flow is set to 0.5 agents/second, corresponding to $[13,14]$.

Injecting new agents to the system is accomplished by dynamic sources. In this way a given number of new agents is created with a defined frequency. Last, to model peoples' movement according to eventual organisational activities in the station, 'waiting areas' (wa) are implemented in JuPedSim and used in the simulations. One waiting area is placed inside the entry hall in front of the tunnel entry $\left(w a_{\text {hall }}\right)$, one is in front of every stair in the tunnel $\left(w a_{\text {tunnel }}\right)$ and one is in front of every train door $\left(w a_{\text {doors }}\right)$. Waiting areas are considered as intermediary goals, as they may be targeted by agents, while evacuating to their final destination (trains). Moreover, they serve as a spot to hold agents for a certain amount of time, before continuing the evacuation process to the next destination. As a side-effect of this waiting mechanism, the number of agents, which is allowed to enter the platforms, can be regulated. In some situations, there will be no train waiting at the platform or its doors are still close. In these cases, people would usually distribute themselves at the platform or in front of the doors to enter the train quickly when it arrives and opens the doors. To model this distribution and waiting of people, the waiting areas in front of the train doors are used. The maximum number of agents in the waiting areas on a platform is defined by the maximum capacity of the train. The other waiting areas in the entry hall and the tunnel can be used to avoid crowding by regulating the inflow and to guide agents to the next goal. This corresponds to the organisational activities of the assigned security personnel.

\begin{tabular}{l|c} 
Type of train & Regional Express (RE) \\
\hline Number of wagons & 9 \\
\hline Number of doors (middle wagons) & 2 \\
\hline Number of doors (end wagons) & 1 \\
\hline Door width & $2 \mathrm{~m}$ \\
\hline Distance between wagon's doors & $18 \mathrm{~m}$ \\
\hline Distance between doors of adjacent wagons & $6 \mathrm{~m}$ \\
\hline
\end{tabular}

Table 1: Technical specifications of the selected train type

In order to investigate the capacity of the station during the evacuation, the initial condition in the simulation depicts an intermediate point in time, when the regular rail traffic has been stopped and trains are just used to evacuate people. Two scenarios for crowd management measures are considered:

1. No organisational activities: people move freely in the station and on the platforms.

2. Organisational activities: free movement is restricted by barriers and security staff. The inflow to the station is regulated and people are distributed accordingly on the platforms, to optimise the entry times into the trains.

To investigate the impact of these variations, several setups are accomplished, see table 2 . The first column shows the scenario and the second shows the different setups, which will be described below. Columns three to six show the varied parameters. The waiting areas are varied 
in their waiting times $(w t)$ and the sources in their frequency $(f)$. The variation in the entrances is the usage of one or two entrances. The specified goals mean whether the agents reach a specific train or any train.

In the first setup (1a) of the first scenario 4400 agents are distributed randomly in the station. Half of them have a defined goal, which leads them to a specific platform. The others do not have a specified goal and take the first train they can reach. As the station has two entrances, one at the entry hall and one at the other end of the tunnel, the agents do not all walk into the same direction in this setup. Some are starting at the end of the tunnel, but target the first platform with its access being at the beginning of the tunnel.

In setup $1 \mathrm{~b}$ the back entrance is closed. So the agents can only enter the station from the entry hall. To estimate critical bottlenecks, 3200 agents are distributed randomly in the entry hall at the beginning of the simulation. From there they walk through the station, $50 \%$ to individual goals (train doors) and $50 \%$ to the first platform and train they can reach.

\begin{tabular}{c|c|c|c|c|c} 
scenario & setup & waiting areas & sources $(f)$ & entrances & specified goals \\
\hline 1 & $1 \mathrm{a}$ & None & None & 2 & yes: $1 / 2$, no: $1 / 2$ \\
\hline 1 & $1 \mathrm{~b}$ & None & None & 1 & yes: $1 / 2$, no: $1 / 2$ \\
\hline 2 & $2 \mathrm{a}$ & $w t_{\text {hall }}: 60 \mathrm{~s}, w t_{\text {tunnel }}: 180 \mathrm{~s}$ & $5 \mathrm{~min}$ & 1 & all \\
\hline 2 & $2 \mathrm{~b}$ & $w t_{\text {hall }}: 60 \mathrm{~s}, w t_{\text {tunnel }}: 180 \mathrm{~s}$ & $10 \mathrm{~min}$ & 1 & all \\
\hline 2 & $2 \mathrm{c}$ & $w t_{\text {hall }}: 60 \mathrm{~s}, w t_{\text {tunnel }}: 0 \mathrm{~s}$ & $10 \mathrm{~min}$ & 1 & all \\
\hline
\end{tabular}

Table 2: Setup varieties of the two scenarios

In the second scenario all agents start in the tunnel at a position next to their destination platform. This corresponds to a starting situation where the agents have entered the station and were distributed in the tunnel to reach their destination platform. Altogether there are 1600 agents at the beginning of the simulation. Additionally, the sources are used to add new agents to the systems four times (sources a - d). With the first source (a) 1600 agents are added. With the other sources $(b-d) 3200$ agents are added, which corresponds to 640 agents per platform or train. To model the operational activity of barriers and security staff, the outlined waiting areas are used.

The parameters of the first setup (2a) of this scenario are shown in table 3 . They are based on a train timing of five minutes. The first column shows the sources' reference number ( $\mathrm{a}$ d). The second shows the start time $\left(t_{0}\right)$ of the sources, where the first people are added in the simulation. The next columns show the end time of the sources $\left(t_{1}\right)$, the frequency ( freq) and the number of agents added per given frequency. The waiting times of the waiting areas are set like shown in columns six to eight.

\begin{tabular}{c|c|c|c|c|c|c|c} 
Source & $t_{0}[\mathrm{~s}]$ & $t_{1}[\mathrm{~s}]$ & freq $[\mathrm{s}]$ & agents $/ f$ & $w t_{\text {hall }}[\mathrm{s}]$ & $w t_{\text {tunnel }}[\mathrm{s}]$ & $w t_{\text {doors }}[\mathrm{s}]$ \\
\hline $\mathrm{a}$ & 0 & 120 & 10 & 160 & 60 & 180 & 360 \\
$\mathrm{~b}$ & 240 & 360 & 20 & 320 & 300 & 420 & 600 \\
$\mathrm{c}$ & 540 & 660 & 20 & 320 & 600 & 720 & 900 \\
$\mathrm{~d}$ & 820 & 960 & 20 & 320 & 900 & 1020 & 1200
\end{tabular}

Table 3: Setup 2a: waiting times and source parameter based on a train timing of 5 minutes

A variation of setup $2 \mathrm{a}$ is done in setup $2 \mathrm{~b}$. Here the intervals between the sources were increased. It is now based on train timing of ten minutes instead of five minutes. Additionally, 
the agents, who start in the tunnel at the beginning of the simulation are arranged closer to the platform accesses.

The last setup variation (2c) is the adaption of the waiting times in the entry hall and in front of the stairs inside the tunnel. As can be seen in table 4 the waiting times in $w a_{\text {hall }}$ and $w a_{\text {tunnel }}$ are now equal. Therefore, the waiting areas inside the tunnel serve only to guide the agents to the next goal, without making them wait in front of the stairs.

\begin{tabular}{c|c|c|c|c|c|c|c} 
Source & $t_{0}[\mathrm{~s}]$ & $t_{1}[\mathrm{~s}]$ & $f r e q[\mathrm{~s}]$ & peds $/ f$ & $w t_{\text {hall }}[\mathrm{s}]$ & $w t_{\text {tunnel }}[\mathrm{s}]$ & $w t_{\text {doors }}[\mathrm{s}]$ \\
\hline 1 & 0 & 150 & 10 & 160 & 60 & 0 & 500 \\
2 & 540 & 690 & 20 & 320 & 600 & 600 & 1100 \\
3 & 1140 & 1290 & 20 & 320 & 1200 & 1200 & 1700 \\
4 & 1720 & 1890 & 20 & 320 & 1800 & 1800 & 2300
\end{tabular}

Table 4: Setup 2c: waiting times and source parameter based on a train timing of 10 minutes

\section{$3 \quad$ Results}

As mentioned before, two different scenarios with setup variations are simulated. Setup 1a, started with a random distribution of agents in the station, especially in the tunnel, which corresponds to the usage of two entrances, see figure 2. In this setup, the tunnel is identified as a critical bottleneck. We observe a bidirectional flow inside the tunnel, which causes huge congestion in its middle, see figure 3 (left). It took about 15 minutes until this congestion has been dissolved. Adding more agents to the station without any regulation would cause larger congestion and crowding.



Figure 2: Simulation of setup 1a at time step $t=0 \mathrm{~s}$.

To avoid a bidirectional flow in the tunnel one entrance should be closed. This is done in the 
second variation of the first scenario (setup 1b). Here the transition between the entry hall and the tunnel as well as the stairs are identified as critical bottlenecks. Figure 3 (right) shows that congestion occurs at the transition between the entry hall and the tunnel. After the agents have passed this transition, further congestion appears in front of the stairs, because the walking speed is slower when walking upstairs than walking on a horizontal corridor. The congestion dissolves approximately after four minutes, which displays a significant progress compared to setup 1a. However, the last agent enters the train after more than seven minutes. As the trains should depart every five minutes, the setup has to be optimised to prevent congestion at any time.
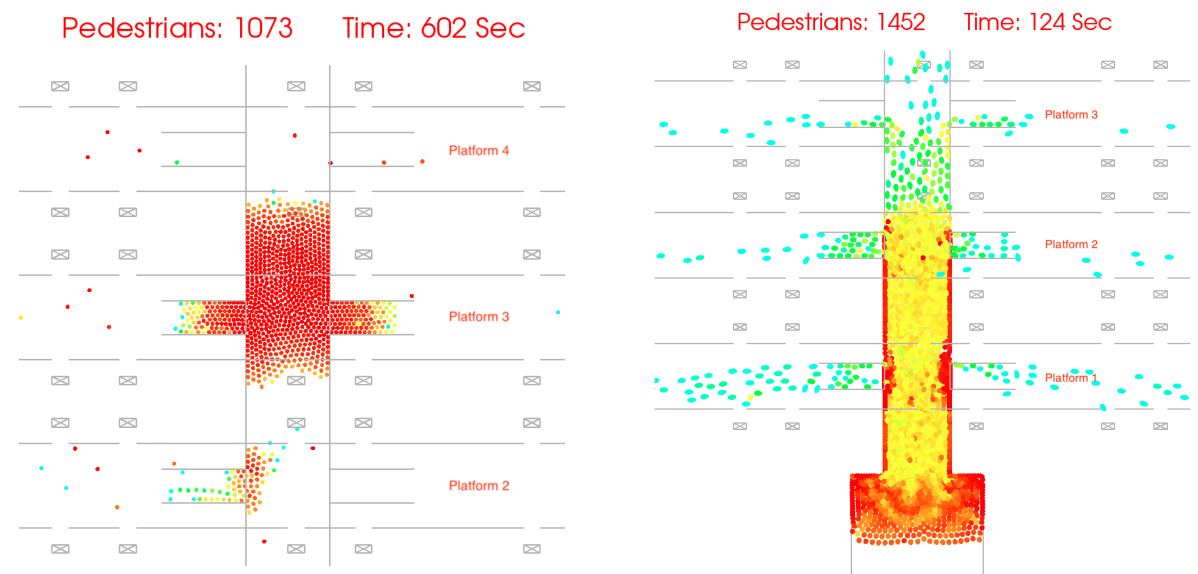

Figure 3: Results of the two setup variations of scenario 1: setup 1a: congestion caused by a bidirectional flow inside the tunnel (left), setup 1b: congestion between the entry hall and the first platform access (right).

Therefore, in the second scenario the inflow is regulated and the agents are guided through the station. Figure 4 shows the results for the three setups. No bidirectional flow is caused in any of the setups, because the agents only start from the entry hall. Nevertheless, the results of setup 2a (figure 4, left) show similarities to those of setup 1b. In this setup the transition between the entry hall and the tunnel is also identified as critical bottleneck. A significant congestion occurs between this transition and the first platform-access inside the tunnel. This prevents agents from reaching the rear platforms. The cause is underestimated waiting times in the entry hall or overestimated waiting times in the tunnel. In addition the sources are identified as a second bottleneck. The interval of the sources is chosen too short. The congestion is not dissolved when new agents were added. Together with the under- and overestimated waiting times, this leads to massive congestion and crowding, which can not be reduced without stopping the inflow. Hence, the trains are not able to comply with their timing of five minutes.

By the inflow restriction in setup $2 \mathrm{~b}$, the congestion, which arises in the area between the entry hall and the first platform-access in the tunnel, can be reduced, see figure 4 (middle). However, as this inflow restriction can still not prevent further tailback when more agents are added constantly to the system, the sources and the transition between the entry hall and the tunnel remain critical bottlenecks. Again this leads to massive congestion and crowding, which can not be reduced without stopping the inflow. Hence, as in both setups (2a and $2 \mathrm{~b}$ ) the flow collapses after a short time, they are not suitable to estimate the maximal capacity of the train 
station in case of a big-scale emergency evacuation with trains.

In the third setup (2c) no critical bottlenecks can be identified. Congestion no longer arises in the area between the entry hall and the beginning of the tunnel, see figure 4 (right). Moreover, after new agents were added to the system, there is still no congestion. In this way, five trains (3200 agents) could be filled every ten minutes, which corresponds to a capacity of approximately 19000 agents per hour. In conclusion, the variations of setup 2c, with waiting areas inside the entry hall in front of the tunnel, but without waiting times in front of the stairs, show the best flow properties and therefore the best capacity.


Figure 4: Results of all setup variations of scenario 2: setup 2a: congestion between the entry hall and the first platform-access (left), setup 2b: congestion between the entry hall and the first platform-access (middle), setup 2c: no congestion (right).

\section{Conclusion and outlook}

Two scenarios with a total of five setup variations have been estimated. The transitions between the entry hall and the tunnel, the stairs and the sources (inflow of agents) were identified as critical bottlenecks in four of the five setups. In setup $2 \mathrm{c}$ the sources and waiting times were based on a train timing of ten minutes. The agents were guided through the station, with waiting times in the entry hall, but without waiting times in the tunnel. In this setup no critical bottlenecks were identified. The results of this setup show the lowest congestion and therefore the highest capacity. With a train timing of ten minutes, the capacity is approximately 19000 agents per hour.

Boarding times also play a significant role. As mentioned before, these were taken from the 
literature and assumed to be 0.5 agents/second in each of the variants described above. These values will be examined by field measurements on running platforms for different types of trains. Furthermore, measurements and surveys will be accomplished at an operational station, where passengers movements and flows at peak times and in individual events are recorded and analysed. These will serve to further validate the results of the simulations.

Additionally the simulation studies will be extended by examining the influence of the variation of the departure intervals of the trains. Therefore the train schedule of an operational station and more train types will be added to the model. Furthermore, information of city councils will be used to model accurate population distributions.

\section{References}

[1] Todd Litman. Lessons from Katrina and Rita. https://www.researchgate.net/ publication/242081750_Lessons_From_Katrina_and_Rita_What_Major_Disasters_Can_Teach_ Transportation_Planners, 2006.

[2] John L. Renne, Thomas W. Sanchez, and Todd Litman. Carless and special needs evacuation planning: A literature review. https://journals.sagepub.com/doi/full/10.1177/ $0885412211412315,2011$.

[3] Presidenza del Consiglio dei Ministri Dipartimento della Protezione Civile Dipartimento Idraulica Trasporti e Strade. Aggiornamento dei Piani di Emergenza per il Rischio Vulcanico Nell'area Vesuviana e Nell'area Flegrea. http://www.protezionecivile.gov.it/jcms/it/view_pde.wp? contentId=PDE12771\#zone_pericolosita, 2006.

[4] G. Rolandi. Volcanic hazard at vesuvius: An analysis for the revision of the current emergency plan. https://www.sciencedirect.com/science/article/abs/pii/S0377027309003254? via\%3Dihub, 2009.

[5] Tullio Ricci, Rosa Nave, and Franco Barberi. Vesuvio civil protection exercise MESIMEX: survey on volcanic risk perception. https://www . annalsofgeophysics.eu/index.php/annals/article/ view/6458, 2012.

[6] AG Fukushima UAG Evakuierungsplanung. Rahmenempfehlungen für die Planung und Durchführung von Evakuierungsmaßnahmen einschließlich der Evakuierung für eine erweiterte Region. https://www.bbk.bund.de/DE/Service/Fachinformationsstelle/ RechtundVorschriften/Rechtsgrundlagen/IMK-Beschluesse/imk-beschluesse_node.html, 2014. TOP 34, Anlage 3.

[7] Armel Ulrich Kemloh Wagoum, Mohcine Chraibi, and Jun Zhang. JuPedSim: An open framework for simulating and analyzing the dynamics of pedestrians. In 3rd Conference of Transportation Research Group of India, 2015.

[8] Flurin Silvan Hänseler. Modeling and estimation of pedestrian flows in train stations. PhD thesis, École Polytechnique Fédérale de Lausanne, 2016.

[9] Schröder, Haensel, Arnold, Chraibi, and Seyfried. Knowledge- and Perception-based Route Choice Modelling in Case of Fire. http://juser.fz-juelich.de/record/255940, 2015.

[10] Mohcine Chraibi. Jülich Pedestrian Simulator - User's Guide Version 0.8.2. Forschungszentrum Juelich GmbH, 2017.

[11] Arne Graf. Automated Routing in Pedestrian Dynamics. Master's thesis, Fachhochschule Aachen, Campus Jülich, 2015.

[12] Antoine Tordeux, Mohcine Chraibi, and Armin Seyfried. Der Fahrgastwechsel im öffentlichen Personenverkehr. https://arxiv.org/pdf/1512.05597.pdf, 2015.

[13] Benjamin Schröder. Grunddaten der Personendynamik in Schienenfahrzeugen. Master's thesis, Bergische Universität Wuppertal, 2013. 
[14] Weidmann. Der Fahrgastwechsel im öffentlichen Personenverkehr. https://www . research-collection.ethz.ch/handle/20.500.11850/47988, 1994. 